Disclosure of Interests: Patrick Durez Speakers bureau: Bristol-Myers Squibb, Eli Lilly, Sanofi, Celltrion, Rene Westhovens Grant/research support from: Bristol-Myers Squibb, Consultant for: Celltrion, GalapagosGilead, Yedid Elbez Employee of: Employee of Excelya which received funding from Bristol-Myers Squibb as contract research organization., Sofie Robert Shareholder of: Bristol-Myers Squibb, Employee of: Bristol-Myers Squibb, Harris A Ahmad Shareholder of: Bristol-Myers Squibb, Employee of: Bristol-Myers Squibb

DOI: 10.1136/annrheumdis-2019-eular.3134

\section{SAT0085 A MATRIX RISK MODEL FOR PREDICTING 5-YEAR RADIOGRAPHIC PROGRESSION IN A COHORT OF EARLY RHEUMATOID ARTHRITIS TREATED ACCORDING TO T2T STRATEGY}

Anna Laura Fedele, Dario Bruno, Barbara Tolusso, Luca Petricca, Gianfranco Ferraccioli, Elisa Gremese. Division of Rheumatology, Fondazione Policlinico Universitario A. Gemelli IRCCS, Rome, Italy

Background: In the treatment strategy of early rheumatoid arthritis (ERA) it is of pivotal importance to detect those patients who are at risk of radiographic progression (RP) in order to avoid decline in both functional capacity and quality of life.

Objectives: To identify baseline predictive factors for 5-year RP in an observational cohort of ERA treated according to treat-to-target (T2T) strategy, and to create a matrix risk model including the strongest predictors.

Methods: A total of 212 ERA patients with less than 12 months of disease duration (mean age $52.8 \pm 13.9$ years, $75 \%$ female, $74.5 \%$ seropositive) and with available radiographs at 5 years of follow-up (FU), were enrolled in the study. ERA patients fulfilled the 2010 ACR criteria for RA and were followed according to the T2T strategy. At baseline, and every three months, the ACR/EULAR core data set variables were recorded. At baseline and every year, hand and foot radiographs were examined according to modified Total Sharp score (mTSS). At each visit, clinical improvement and remission were evaluated according to EULAR criteria. The achievement of Comprehensive Disease Control (CDC) (28-joint Disease Activity Score using $C$ reactive protein <2.6, Health Assessment Questionnaire $<0.5$ and change from baseline in mTSS $\leq 0.5$ ) was assessed every year of FU.

Results: Fifty-six ERA patients $(26.4 \%)$ had erosions on hand and foot radiographs performed at baseline.

At 5 years of FU additional 56 subjects $(26.4 \%$ ) developed RP. ERA patients showing RP were in higher percentage ACPA positive $(78.6 \%)$ and erosive at baseline $(41.1 \%)$ compared to subjects not manifesting RP (65.4\% ACPA positive, $p=0.07 ; 21.2 \%$ erosive at baseline, $p=0.004$, respectively). Moreover, radiographic progressors had a VERA disease (disease duration less than three months) with lower frequency $(21.4 \%)$ compared to non progressors $(34.6 \%, p=0.07)$. CDC was achieved at twelve months of FU more frequently by non progressors $(52.6 \%)$ compared to patients developing $\operatorname{RP}(30.4 \%, p=0.004)$. There were no differences regarding age, disease duration, BMI and smoking habit. Comparing the treatments between the two groups, we found similar frequencies of conventional and biotechnological drugs.

In logistic regression analysis, the variables predictive of 5-year RP were ACPA positivity [OR $(95 \% \mathrm{Cl}): 2.51(1.12-5.63)]$, presence of erosions at baseline [OR $(95 \% \mathrm{Cl}): 2.42(1.16-5.06)]$ and not reaching $\mathrm{CDC}$ at the twelfth month of FU [OR $(95 \% \mathrm{Cl}): 2.38(1.18-4.76)]$. Combining the coexistence of erosions at baseline and ACPA positivity (registered in 35 subjects, $16.5 \% \mathrm{O}$ of the total cohort), the risk of RP increased [OR $(95 \% \mathrm{Cl})$ : $3.50(1.55-7.88)]$.

Dividing patients according to autoantibody positivity, the independent variables associated to RP were the presence of erosions at baseline [OR $(95 \% \mathrm{Cl}): 2.91(1.28-6.63)]$ and not having a VERA disease [OR $(95 \% \mathrm{Cl})$ : $2.86(1.06-7.69)]$. In seronegative subjects predictive factors of RP were not identified.

Using the parameters showing the strongest association with RP, threeparameter risk matrix was created, showing the proportion of ERA patients with RP in each box (Figure 1).

The higher proportion of RP was showed in ACPA positive patients, regardless of all the other baseline predictors.

Conclusion: In our cohort the predictive factors of 5-year RP were ACPA positivity, presence of erosions at baseline and not achieving CDC at $12^{\text {th }}$ of FU. Although CDC is the advisable treatment goal, the radiological damage is not fully prevented in case of ACPA positivity.

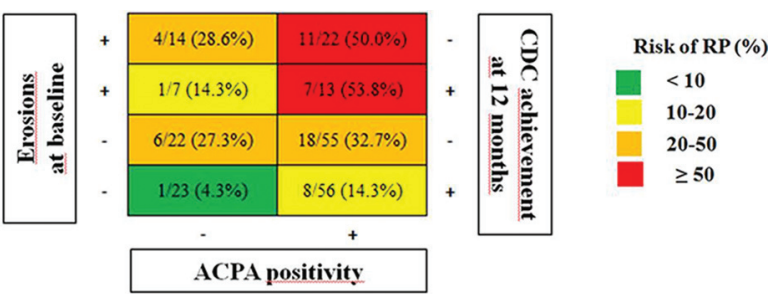

Figure 1. Three-parameters risk matrix showing the proportion of ERA patients with RP.

Disclosure of Interests: Anna Laura Fedele: None declared, Dario Bruno: None declared, Barbara Tolusso: None declared, Luca Petricca: None declared, Gianfranco Ferraccioli Speakers bureau: BMS, Roche, Elisa Gremese Consultant for: AbbVie, BMS, Celgene, Janssen, Lilly, MSD, Novartis, Sanofi, UCB, Roche, and Pfizer, Speakers bureau: BMS, Speakers bureau: Roche, Speakers bureau: AbbVie, BMS, Celgene, Janssen, Lilly, MSD, Novartis, Sanofi, UCB, Roche, and Pfizer DOI: 10.1136/annrheumdis-2019-eular.6372

\section{SAT0086 EVALUATION OF MEDICATION PERSISTENCE IN PATIENTS WITH RHEUMATOID ARTHRITIS TREATED WITH NON-TNFI DISEASE-MODIFYING ANTI-RHEUMATIC DRUGS}

Leticia Ferri ${ }^{1}$, Evo Alemao ${ }^{1}$, Sonie Lama ${ }^{1}$, Aarti Rao ${ }^{2} .{ }^{1}$ Bristol-Myers Squibb, Princeton, United States of America; ${ }^{2}$ Mu Sigma, Bangalore, India

Background: Persistence to prescribed biologic (b)DMARDs, such as TNF inhibitors (TNFi), in patients (pts) with RA has been suboptimal, with rates ranging from $30-80 \%$. There are limited data on the persistence of non-TNFi bDMARDs and targeted synthetic DMARDs, such as tofacitinib (TOF).

Objectives: To compare medication persistence in pts with RA treated with different non-TNFi DMARD treatment options including abatacep (ABA), TOF and tocilizumab (TOC), and to evaluate characteristics (e.g. demographics, comorbidities, baseline treatments) of these pts.

Methods: Pts ( $\geq 18$ years) from the Truven MarketScan ${ }^{\mathrm{TM}}$ administrative claims database with RA ( $>2$ claims for RA identified with International Classification of Diseases [ICD]-9 or ICD-10) being treated with a nonTNFi DMARD from Jan 2006 to Sep 2017 were included. The date of the first claim of a drug of interest on or after diagnosis of RA was considered the index date. Pts were required to have at least 6 months of continuous enrolment prior to the index date (baseline). Pts with claims of other bDMARDs at the index date were excluded from the study. Persistence was defined as the number of days from the index date to the first switch or discontinuation of the index date DMARD, or end of the study period, or end of enrolment, whichever came first. Pts were stratified by prior TNFi use (TNFi-naïve or TNFi-experienced) and by prior corticosteroid (CS) use. Pairwise comparison of persistence between the non-TNFi DMARDs was performed, with $A B A$ as the reference using Kruskal-Wallis test. A $p$ value of $<0.05$ was considered statistically significant.

Results: A total of 30,556 pts satisfied the inclusion and exclusion criteria, of which $20,410,5048$ and 5098 were initially treated with ABA, TOF and TOC, respectively. Overall, the ages of therapy initiation for the ABA and TOF cohorts were comparable, while the TOC cohort was slightly younger. The majority $(80 \%)$ of the study population was female. The proportion of pts with prior CS use was lower in the ABA cohort vs the other cohorts. Overall, the comorbidity index between treatment cohorts was similar; however, there were some differences in types of comorbidities at baseline (Table 1). The ABA cohort tended to have a lower proportion of pts with chronic obstructive pulmonary disease/asthma, pulmonary nodule, dyslipidaemia and osteoarthritis, while the proportions of pts with heart failure, ischemic heart disease and lupus were higher relative to other cohorts (Table 1). Overall, mean (SD) time on therapy for pts in the ABA cohort was longer compared with pts in the TOF (338 [418] vs 257 [288] days) and the TOC cohorts (338 [418] vs 294 [345] days). Similar differences were observed when further stratified by pts' prior use of TNF and CS (Table 2).

Conclusion: Based on the analysis of a large US claims database, pts with RA who were prescribed abatacept had higher persistency with therapy compared with other non-TNFi DMARDs such as TOF and TOC, regardless of prior use of $\mathrm{TNFi}$ and CS. Further analysis of medication persistence adjusting for pt characteristics is warranted. 